\title{
In reply: Sevoflurane or desflurane: Which one is more expensive? Limiting the accessibility of cost-prohibitive drugs: The story is incomplete
}

\author{
Ariana K. Tabing, MD, MBA (1) • Jesse M. Ehrenfeld, MD, MPH • \\ Jonathan P. Wanderer, MD, MPhil
}

Received: 29 October 2015/Accepted: 3 November 2015/Published online: 17 December 2015

(c) Canadian Anesthesiologists' Society 2015

\section{To the Editor,}

We greatly appreciate the two thoughtful replies to our recent publication. Both De Medts et al. ${ }^{1}$ and McConachie et al. ${ }^{2}$ inquired about our average fresh gas flow rates as well as our use of nitrous oxide $\left(\mathrm{N}_{2} \mathrm{O}\right)$. Anesthesia providers at our institution predominantly use $1 \mathrm{~L} \cdot \mathrm{min}^{-1}$ air combined with $1 \mathrm{~L} \cdot \mathrm{min}^{-1}$ oxygen for anesthetic maintenance without significant use of $\mathrm{N}_{2} \mathrm{O}$. We look forward to the studies in progress detailing the benefits of using $\mathrm{N}_{2} \mathrm{O}$ combined with desflurane. Additionally, we agree that low-flow techniques have been shown to result in cost savings, as referenced in our discussion, and we appreciate that they may provide another modality for limiting costs while preserving quality of care. Our institution currently has more traditional anesthesia machines that do not support automated lowflow approaches.

To address the point by De Medts et al. about the synergistic use of opioids, our institution has been transitioning away from an approach that relies heavily on opioids towards one that uses multimodal analgesia for a

A. K. Tabing, MD, MBA ( $\square)$

Vanderbilt University School of Medicine, Vanderbilt

University, Nashville, TN, USA

e-mail: Ariana.tabing@gmail.com

J. M. Ehrenfeld, MD, MPH · J. P. Wanderer, MD, MPhil Departments of Anesthesiology, Vanderbilt University, Nashville, TN, USA

J. M. Ehrenfeld, MD, MPH · J. P. Wanderer, MD, MPhil Biomedical Informatics, Vanderbilt University, Nashville, TN, USA

J. M. Ehrenfeld, MD, MPH

Health Policy and Surgery, Vanderbilt University, Nashville, TN, USA reduction in postoperative opioid-related side effects. With regard to the inquiry by McConachie et al. about ambulatory cases, approximately one-third of the surgical cases in the time period and locations of our study can be classified as same-day surgeries. The corrected difference between the average case costs of desflurane and sevoflurane is addressed in another response in the Journal titled, "In reply: Observational study of prolonged times to tracheal extubation". ${ }^{3}$ Finally, in our view, our study serves to show that significant cost savings can be achieved without the large capital investment that would be required to deploy newer more sophisticated machines. Just as there are many appropriate techniques for providing anesthesia, there are likely many suitable algorithms to limit costs in anesthesia practices. We intended to show that small changes in protocol could be effective in producing significant cost reduction without a decrease in quality of care.

Acknowledgments Dr. Wanderer is supported by the Foundation for Anesthesia Education and Research (FAER) and the Anesthesia Quality Institute (AQI), Mentored Research Training Grant in Health Services Research (MRTG-HSR).

Conflicts of interest None declared.

\section{References}

1. De Medts R, Hendrickx JF, De Wolf AM, Carette R. Sevoflurane or desflurane: Which one is more expensive? Can J Anesth 2016; 63: this issue. DOI:10.1007/s12630-015-0530-9.

2. McConachie I. Limiting the accessibility of cost-prohibitive drugs: The story is incomplete. Can J Anesth 2016; 63: this issue. DOI:10.1007/s12630-015-0531-8.

3. Tabing AK, Ehrenfeld JM, Wanderer JP. In reply: Observational study of prolonged times to tracheal extubation. Can J Anesth 2016. DOI:10.1007/s12630-015-0497-6. 